\title{
El hecho educativo desde la perspectiva y experiencia de una maestra "normal"
}

\author{
The educational fact from the perspective and experience of a "normal" teacher
}

María Dolores Sancho

Universidad Nacional del Comahue - Argentina

Neuquén, Argentina

maria.sancho@fade.uncoma.edu.ar

\section{RESUMEN}

Dada la importancia que han tenido las escuelas normales en la historia argentina, el presente artículo tiene como objetivo analizar el relato de una maestra formada en dichas escuelas -una maestra "normal"- acerca del hecho educativo a partir de las principales perspectivas teóricas de la sociología de la educación y, con el fin, de determinar qué tipo de enfoque predomina en este caso concreto. La metodología empleada para tal fin consiste en el análisis de una carta escrita por ella donde cuenta su experiencia como docente entre 1966 y 1979. Se trata de una docente que se recibió a mediados de la década del cuarenta en una escuela normal dependiente de la primera escuela normal del país fundada por Domingo Faustino Sarmiento, a saber, la Escuela Normal de Paraná, creada por ley en 1869. Esta carta es analizada desde los enfoques teóricos más importantes de la sociología de la educación como el funcionalismo, el crítico-reproductivismo, el interaccionismo simbólico, la pedagogía crítica y la teoría de la resistencia. A modo de conclusión, se puede decir que, más allá de que existen contradicciones en la experiencia, en el relato de la docente se encuentra una visión del hecho educativo más cercana a la perspectiva funcionalista que a cualquier otra que impacta en sus prácticas.

Palabras clave: Hecho educativo; Escuela Normal; Sociología de la Educación; Funcionalismo

\begin{abstract}
Given the importance that normal schools have had in Argentine history, this article aims to analyze the story of a teacher trained in these schools -a "normal" teacher - about the educational fact from the main theoretical perspectives of the sociology of education and, in order to determine what kind of approach prevails in this specific case. The methodology used for this purpose consists in the analysis of a letter written by her where she talks about her experience as a teacher between 1966 and 1979. It is about a teacher who was received in the mid-1940s in a normal school that depended on the first normal school in the country founded by Domingo Faustino Sarmiento, namely the Normal School of Paraná, created by law in 1869. This letter is analyzed from the most important theoretical approaches to the sociology of education such as functionalism, critical-reproductivism, symbolic interactionism, critical pedagogy and resistance theory. By way of conclusion, it can be said that, beyond the fact that there are contradictions in the experience, in the teacher's account there is a vision of the educational fact that is closer to the functionalist perspective than to any other that impacts her practices.
\end{abstract}

Keywords: Educational fact; Normal school; Sociology of education; Functionalism 


\section{INTRODUCCIÓN}

En Argentina, el escaso desarrollo socio-económico llevó a que sea la propia estructuración del Estado -desarrollada entre 1862 y 1880- la que genere las bases de la unidad nacional enmarcada, por un lado, en una identidad colectiva (faceta simbólica) y, por el otro, en la consolidación de una estructura de clases, dentro de la cual la burguesía agraria cumplirá un rol dirigente (Ouviña, 2002). A partir de esto, y en consonancia con lo que sucedió en otros países de América Latina, emergió una forma de estado particular: el Estado Oligárquico. A grandes rasgos, se puede decir que una de las características centrales de éste es que se encuentra fuertemente ligado a los intereses latifundistas y agroexportadores de un reducido sector social que controla el principal recurso económico en la región, o sea la producción de materias primas. En este marco, la prioridad del aparato estatal fue privilegiar la garantía del "orden" a costa de los derechos políticos de la mayoría de la población, o sea que respaldaba las libertades civiles, pero limitaba las libertades políticas, en la búsqueda de un marco propicio para el desarrollo económico identificado con el "progreso" capitalista (Ouviña, 2002).

En este marco, se creó un sistema educativo organizado y monopolizado por el Estado con el fin de lograr la integración y modernización social, así como también se sancionó la Ley de Educación Común № 1.420 que fijó el carácter obligatorio, gratuito, gradual y laico de la educación primaria (Altamirano, 2018). Así, la educación se propuso inculcarles a los individuos los conocimientos necesarios para incorporarse al mundo social y para constituir una identidad nacional inexistente. Para cumplir con esta tarea, fue necesario contar con recursos humanos capacitados: así se crearon las escuelas normales, como instituciones oficiales nacionales, cuyo objetivo era de capacitar a los maestros que, luego, ejercerían la función de enseñar, en el marco de la instrucción pública, a las nuevas generaciones (Altamirano, 2018).

A partir de lo dicho anteriormente, se puede dimensionar la importancia que han tenido las escuelas normales en la historia argentina, específicamente, en la construcción de la identidad nacional. Y aquí se encuentra el "motor" del presente artículo: analizar la experiencia de una maestra formada en una escuela normal para capacitar a nuevas generaciones en una Argentina carente de unidad nacional. Se trata de una docente, a quién llamaremos María, que se recibió a mediados de la década del cuarenta en una escuela normal dependiente de la primera escuela normal del país fundada por Domingo Faustino Sarmiento, a saber, la Escuela Normal de Paraná, creada por ley en 1869. Esta maestra ejerció su labor durante 33 años. Su relato se centra en su experiencia como docente en una escuela ubicada en un barrio popular de la ciudad de Alta Gracia, Provincia de Córdoba - Argentina, entre 1966 y 1979.

En este sentido, el objetivo del siguiente artículo es analizar el relato de una maestra "normal" acerca del hecho educativo a partir de distintas perspectivas teóricas de la sociología de la educación con el fin de determinar qué tipo de enfoque predomina. Para este fin, se analizó una carta escrita por dicha maestra acerca de los rasgos más destacados de su experiencia como docente y se seleccionaron algunos fragmentos de la misma en función de su relación los enfoques teóricos más importantes de la sociología de la educación como el funcionalismo (Durkheim, Parsons, Dreeben), el crítico-reproductivismo (Bourdieu y Passeron), el interaccionismo simbólico (Becker, Goffman, Rist), la pedagogía crítica y la teoría de la resistencia (Freire, Ranciere, Giroux, Aronowitz).

\section{METODOLOGÍA}

Este relato da cuenta de varios aspectos de su experiencia docente. Así, por ejemplo, cuenta que, en esa escuela, era necesario desempeñarse como maestra y como "asistente social" por lo que cuando comenzaba el año ella iba a cada una de las casas de sus alumnos/as a conocer a sus familias, donde y como vivían. Asimismo, relata que los niños/as que menos afecto recibían, eran los que daban mas trabajo, por lo que ella encontraba en el afecto su primera arma para enseñar. Además, cuenta como hacía para enseñar geometría ante la imposibilidad material de acceder a los útiles necesarios para ello como por ejemplo el compás. También relata como en una "salida de campo", donde se pusieron a ver como el hornero construía su nido, se llevó la sorpresa de que los estudiantes sabían más del hornero que ella. Por 
último, comenta que eligió enseñar "huerta” porque consideraba que era muy útil para los niños/as que podían hacerla en sus casas.

\section{DESARROLLO}

\subsection{Una misma experiencia, distintas perspectivas}

Como se sostuvo anteriormente, el relato de María sobre su experiencia como docente en una escuela ubicada en un barrio popular de la ciudad de Alta Gracia, Provincia de Córdoba - Argentina, entre 1966 y 1979, puede ser analizado desde distintas perspectivas sociológicas acerca de la educación. Esto da cuenta de las diferentes tradiciones que se cruzan en este caso concreto que no son coherentes sino más bien contradictorias. Así, en primer lugar, este puede ser leído desde la perspectiva de la sociología clásica y el funcionalismo. Desde el punto de vista de la sociología clásica, específicamente de Emile Durkheim (1858-1917), la educación es un hecho social en tanto es algo que se impone a los individuos con una fuerza irresistible que se manifiesta en el momento en que se intenta resistir a ella. En términos de Durkheim,

\footnotetext{
...un hecho social es toda manera de hacer, establecida o no, que puede ejercer sobre el individuo una imposición exterior; o también, que es general en la extensión de una sociedad dada, al mismo tiempo que tiene una existencia propia, independiente de sus manifestaciones individuales (2003, p. 36).
}

Dado que Durkheim estaba preocupado por el tema de la cohesión social se pregunta como es posible producir individuos que al mismo tiempo no destruyan el orden social, o sea que sean cada vez más "individuos" y, al mismo tiempo, estén cada vez más unidos al cuerpo social. La respuesta la encuentra en la educación. De acuerdo con Durkheim, una condición indispensable para la existencia de la ésta es la presencia de una generación de adultos y otra de jóvenes y que los primeros ejerzan una acción sobre los segundos que consista en la inculcación de sentimientos, pensamientos, ideas, conocimientos, prácticas, tradiciones, costumbres, disciplina, autoridad, etc. con el fin de lograr cierta homogeneidad y, de este modo, la existencia de la sociedad.

Esto se relaciona con la concepción de individuo que tiene el autor. Según Durkheim, lo propio de los individuos es proponerse deseos ilimitados que no pueden ser satisfechos con sus medios es decir "puesto que nada los limita, rebasan siempre infinitamente los medios de los que disponen; nada por tanto podrá calmarlos" (Durkheim, 2008, p. 46). Esta situación condena a los hombres a un estado constante de insatisfacción. Sin embargo, para evitar esta situación, es necesario que las pasiones sean limitadas por alguna fuerza exterior que respeten y a la cual se sometan espontáneamente: aquí es donde la sociedad, la educación y las reglas juegan su rol moral.

Esta concepción de la educación se puede ver en el relato de la maestra. Así, por ejemplo, en la carta cuenta que cuando tenía que enseñarles geometría, específicamente circunferencia y círculo, recurría a piolas y clavitos con el fin de fabricar sus propios compases y la clase era dada en el parque del barrio. Ella preparaba uno grande con un clavo que se pudiera enterrar en tierra y otro para hacer girar la piola y hacían una gran circunferencia. Los niños/as formaban filas luego de la explicación de contenido y pasaban a aplicar los distintos conceptos. Cuenta que eran clases muy divertidas y que los chicos/as no se "indisciplinaban". En este último punto, se observa como, dada su formación "normalizadora", lograr la disciplina en los estudiantes era un objetivo muy importante.

Esta definición esta muy presente en las concepciones actuales acerca de la educación. En este sentido, se encuentra a Gary Fenstermacher quien define a la enseñanza como "una actividad en la que debe haber al menos dos personas, una de las cuales posee un conocimiento o una habilidad que la otra no posee; la primera intenta transmitir estos conocimientos o habilidades a la segunda, estableciéndose entre ambas 
una cierta relación a fin de que la segunda los adquiera" (Gvirtz y Plamidessi, 1998, p. 134). Coincidiendo con el planteo de Gvirtz y Palamidessi (1998) no toda enseñanza va a garantizar un aprendizaje, o sea que el docente enseñe no asegura que sus alumnos/as aprendan, y si aprenden puede pasar que no sea lo que el docente se propuso enseñar.

De acuerdo con Fenstermacher (1989), la enseñanza y el aprendizaje mantienen una relación de dependencia ontológica lo que implica que para que exista la enseñanza debe haber alguien que quiera aprender, es decir que la enseñanza por si sola no existe, a diferencia del aprendizaje que sí se puede dar sin que alguien le enseñe a otro algo. En los fragmentos que hablan de la enseñanza de la geometría, la fauna y la huerta, se visualiza esto ya que en los tres casos hay una docente, un adulto, que intenta enseñarles algo a sus alumnos o sea que existe una situación de enseñanza, aunque no hay garantías de que esos alumnos hayan aprendido lo que la docente quiso enseñar que se observa cuando relata que "los niños/as formaban filas luego de la explicación de contenido y pasaban a aplicar los distintos conceptos" (María, maestra). Aquí puede verse la dependencia ontológica porque sin alumnos que vayan a aprender la docente nunca hubiera podido enseñar.

Dentro del funcionalismo norteamericano, se encuentra a Talcott Parsons (1902-1979) quien recoge los postulados de Durkheim. Este autor concibe a la sociedad moderna como un sistema de roles y, por lo tanto, a la educación como un modo de preparar a los individuos para el desempeño de roles que contribuyen a la armonía social. De este modo, la especificidad de la sociedad moderna se puede encontrar en la educación que se diferencia de la familia en tanto representante de la sociedad tradicional. Mientras que en la primera predomina la universalidad de las normas -iguales para todos e impersonales-, la racionalidad instrumental, el criterio de la adquisición o el resultado en la determinación de la posición social, la recompensa al logro o al merito y los roles específicos, en la segunda prevalece el particularismo de las normas -cambiantes y arbitrarias-, la afectividad, la determinación del estatus del individuo a partir de la adscripción (nacimiento, linaje, familia, edad, sexo, etc.), la recompensa a la necesidad mas allá de las capacidades demostradas y los roles difusos.

De este modo, la escuela es una institución de socialización "secundaria" en donde se busca que los individuos internalicen patrones de comportamiento universalistas-instrumentalistas-orientados al logro-a la adquisición de recompensas de manera separada a otros roles personales. En términos de Robert Drebeen, la escuela les proporciona a los individuos experiencias únicas que, difícilmente, puedan ser adquiridas en otros ámbitos y que conducen a la adquisición de normas de independencia (capacidad de actuar por sí mismos, aceptar la responsabilidad por el propio comportamiento, desempeñar tareas para las que, en ciertas condiciones, se puede esperar la ayuda de los demás), logro (cumplir tareas activamente de la mejor forma posible y controlar el entorno a partir de ciertos criterios de capacidad), universalismo (tratar a los demás como miembros de categorías) y especificidad (tratarlos como miembros de categorías sobre la base de características discretas de la personalidad) (Dreeben, 1990).

Desde este punto de vista, en el relato de la maestra vemos como esta función de la educación se desdibuja ya que no logra socializar al individuo en algunos de esos valores es decir que permanece "tradicionalista" en tanto favorece el particularismo, la afectividad y la recompensa a la necesidad. Esto se observa cuando la maestra cuenta que

\footnotetext{
“empezaba el año escolar y mi primera meta era conocer donde y como vivían mis alumnos (...) cuando yo ya conocía de donde venia ese niño podría tener para cada uno, una forma de hacerle llegar el conocimiento" (María, docente).
}

Asimismo, sostiene que los niños/as que menos afecto recibían, eran los que daban mas trabajo en la "disciplina” por lo que ella encontraba en el afecto su primera arma para enseñar. Cuenta que pasaba por los bancos y acariciaba con su mano las "cabecitas" de sus alumnos/as y ellos/as le levantaban sus 
“ojitos" y le sonreían. Comenta que después de este intercambio todo se le hacia más fácil. Además hace referencia al hecho de sabía que la mayoría de los niños/as sufrían con sus padres ebrios y que trataba de conformarlos cuando éstos los hacían dormir afuera de su casa o les rompían sus tareas para la escuela. Convencida afirma que esto de brindarle afecto y tener en cuenta las historias personales de los niños/as la ayudaba en la forma de hacerles llegar el conocimiento. También es en esta parte del relato donde cuenta que las madres de sus alumnos le confiaban muchos de sus sufrimientos.

Sin embargo, las normas de independencia y logro de Dreeben y el criterio del resultado para determinar la posición social de Parsons, pueden verse en el siguiente extracto del relato donde la maestra remite al trabajo en grupo:

\begin{abstract}
“Aproximadamente 8 mesas con 5 alumnos cada uno. Cuando me interioricé del método me encantó; lo puse en práctica 2 años, pero no me dejaron satisfecha los resultados. Trabajaba el jefe del equipo (que siempre se elige el más capaz) y los demás...casi nada. Aprendían, pero no por lo que investigaba cada uno sino por lo que hacía el más capaz y los demás copiaban. El jefe del grupo quería que su labor se le tuviera en cuenta a él solo y de mala gana lo transmitía (...)" (María, docente).
\end{abstract}

No obstante, desde la perspectiva crítica-reproductivista, estos valores y normas que la escuela asignaría a la/os estudiantes puede ser entendida como "violencia simbólica". Bajo la influencia del marxismo y buscando superar la tensión entre objetivismo y subjetivismo, Pierre Bourdieu y Jean Claude Passeron plantean que la educación no es ajena a los procesos de reproducción social y, por esto, toda acción pedagógica es una imposición que oculta-invisibiliza-naturaliza la dominación de una clase sobre la otra agregándole un plus simbólico a la misma. En este sentido, afirman que

...todo poder de violencia simbólica, o sea, todo poder que logra imponer significaciones e imponerlas como legítimas, disimulando las relaciones de fuerza en que se funda su propia fuerza, añade su fuerza propia, es decir, propiamente simbólica, a esas relaciones de fuerza (Bourdieu y Passeron, 1979, p. 3).

De acuerdo con estos autores, la educación o la acción pedagógica es una violencia simbólica en tanto es la imposición arbitraria de un arbitrario cultural que requiere para su ejercicio de una autoridad pedagógica, que aparece con un derecho de imposición legítima, y de la autonomía relativa de la instancia encargada de ejercerla (Bourdieu y Passeron, 1979). De este modo, aquellos valores que el funcionalismo consagra como universales en realidad son valores particulares de una clase social-dominante- que pretenden ser impuestos como universales a demás clases -dominadas- con el fin de reproducir la estructura social.

Esta acción pedagógica implica, según los autores, la producción de un habitus a partir de la interiorización duradera de los principios de la arbitrariedad cultural de una clase particular permitiendo generar pensamientos y acciones a fines a los mismos una vez finalizada aquella acción pedagógica. De acuerdo con Bourdieu, el habitus se define como un sistema de disposiciones duraderas y trasladables mentales y corporales, adquiridas en el proceso de socialización, que funcionan como principios inconscientes de percepción, apreciación y acción y que están relacionadas a la posición objetiva de los agentes en la estructura social. Son estructuras estructuradas -es producto de condicionamientos objetivos- y estructurantes -organiza las prácticas y contribuye a la reproducción de las estructuras (Bourdieu y Wacquant, 2008). En otras palabras, el habitus se puede definir como la interiorización de posiciones objetivas en forma de disposiciones subjetivas que generan tomas de posición.

El habitus tiene tres atributos: permanencia o durabilidad, exhaustividad o capacidad de reproducir de forma completa los principios de la arbitrariedad cultural en las prácticas, y transferibilidad, o capacidad 
de engendrar practicas conformes a estos principios en el mayor número posible de campos diferentes. Asimismo, existen dos tipos de habitus que, en realidad, forman uno solo al acomodarse: el primario, que corresponde a la socialización primaria y que en general se realiza en el seno de la familia, sobre el cual se constituye el secundario, relacionado a la socialización secundaria, usualmente a cargo de la institución escolar. En este sentido, el éxito en la educación escolar depende de su grado de asimilación con el habitus primario: para las clases dominantes la educación es una continuación del habitus primario mientras que para las clases dominadas la educación se enfrente al habitus primario, o sea que "de allí que la educación escolar que para algunos (los hijos de las clases dominantes) tenga el sentido de una reducación, y para otros (los hijos de las clases dominadas) sea mas bien una deculturación" (Tenti Fanfani, 1994, p. 10).

La formación de habitus secundario a través de la acción pedagógica se ve en el ejemplo antes citado donde la maestra les asigna una tarea grupal a sus estudiantes, que es realizada solo por "los más capaces", donde estos, al ver que sus compañeros no colaboraban en la misma, exigían que "se les reconozca su labor" dando cuenta de la interiorización duradera de los arbitrarios culturales de una clase social. Asimismo, en el relato se encuentra otro ejemplo donde la maestra, en tanto autoridad pedagógica autorizada para impartir conocimientos, les impone a sus alumnos aquello que "deben saber" generando una "deculturación", a partir del uso de diarios como recurso didáctico:

\begin{abstract}
"camino a la escuela, un diariero me regalaba el diario del día anterior. Yo marcaba en cada hoja un artículo que podía ser de interés y los entregaba en las mesas. Lo leía y primero buscaban de qué lugar era la noticia, debían ubicarlo en el mapamundi o en un mapa de donde fuera la noticia. Me preguntaban cuando no entendían algo pero siempre recibieron muy bien esta novedad. Los padres (algunos de los que se acercaban a la escuela que lamentablemente o eran muchos) me comentaban que el hijo les había contado el resumen de la noticia que habían leído ese día. Luego pasaban algunos y daban la crónica con sus palabras a todos los compañeros. Esto favorecía mucho la expresión oral y el conocimiento. Las noticias que yo marcaba eran generalmente de geografía, de investigación científica, de catástrofes climáticas y a veces de deportes. También problemas limítrofes (...)" (María, docente).
\end{abstract}

A partir de este carácter "impositivo" de la educación impartida por María, el relato que estamos analizando puede leerse en el marco de una "pedagogía de los opresores" o del principio del embrutecimiento (Ranciere, 2007) ya que, en términos de Paulo Freire (1921-1997), reifican, o sea deshumanizan, a los estudiantes, a los oprimidos, al estado de objeto pasivo producido por el docente, el opresor, que aparece como su "liberador" cuando en realidad le niega sus posibilidades de ser más, su libertad, su capacidades y su conocimiento, o sea instaura la violencia en la relación, para convencerlos de su superioridad. Esta crítica es propia de la pedagogía crítica y de la teoría de la resistencia que rompen con los planteos funcionalistas acerca de la educación como una relación entre alguien que sabe, que tiene las "respuestas", y alguien que no sabe, que tiene los "problemas".

Sin embargo, también se observan rasgos de la pedagogía del oprimido o de la emancipación (Ranciere, 2007) lo que indica cierta tensión entre ambas. Con este concepto Freire alude a una pedagogía construida con los oprimidos y no para los oprimidos lo que implica reconocer que aquellos antes "oprimidos" tienen saberes, capacidades, pensamientos y sentimientos propios que hacen que la educación implique compartir y elaborar saberes en común. Se trata de una pedagogía que, en términos de Jacques Ranciere (2007), reconoce las capacidades y conocimientos de los "ignorantes" y busca reforzar y desarrollar las mismas. Así, la educación aparece como una praxis -reflexión y acción- liberadora y humanizadora donde no solo se liberan los oprimidos sino también los opresores a partir de la labor de los primeros que en este camino, en primera instancia, deben reconocer que "alojan" al opresor en su interior como modelo, algo que muchas veces se manifiesta como fatalismo - una aparente docilidad-. Esta "doble" liberación se produce en tanto los opresores al negarle a los otros la posibilidad de ser, no pueden a su vez ser, razón por la cual cuando se les quita su poder de oprimir, se les devuelve su humanidad. 
Dentro de esta pedagogía, la dialogicidad, en cuanto encuentro horizontal de los hombres con el objetivo común de saber y actuar, de ser más, es un elemento central de dicha praxis liberadora donde la humildad, el amor, la fe en el poder de hacer y rehacer de los hombres, la confianza y la esperanza son sus condiciones fundamentales. De acuerdo con el autor, esta dialogicidad debe comenzar antes de encontrarse el educador-educando con los educando-educadores es decir cuando aquel se pregunta por el contenido del dialogo ya que este surge a partir de la sistematización de aquellos temas significativos o de la visión sobre su situación existencial concreta planteada por los educando-educadores de forma desordenada y difusa. Así, esta dialogicidad se opone al antidiálogo en tanto supone esloganización, verticalidad, comunicados, imposición, "depositar ideas de un sujeto a otro", seguimiento de un programa.

Así, en el relato se puede observar que la docente no sigue estrictamente el currículum, sino que en muchos casos tiene en cuenta los conocimientos que los estudiantes tienen para elaborar sus clases así como también tiene las condiciones materiales de existencia de los mismos en su planificación. A este respecto, es significativo el siguiente ejemplo:

\begin{abstract}
“es tan pintoresco el lugar donde está la escuela que resolví llevarlos a tres cuadras donde corre el arroyo Alta Gracia. Nos instalamos bajo los arboles a ver la forma como hacía el nido el hornero. Me llevé la sorpresa de que mis alumnos sabían más del hornero que yo (...). Uno de los alumnos me dijo -¿sabe señorita que los horneros no trabajan el día domingo? Me reí, pero los otros chicos me decían lo mismo, así que investigué entre otras personas y me dijeron que era así ¿̇habrá que creer?" (María, docente).
\end{abstract}

En este fragmento se observa que si bien tiene en cuenta que los educando-educadores tienen cosas para enseñar, es decir que son sujetos portadores de conocimientos, también "desconfía” un poco del mismo dada su formación "normalizadora" donde se parte de considerar que existen dos polos, donde uno sabe y el otro no.

A continuación, cuenta que al regresar de dicha salida de "campo" tenían clase de lengua:

\footnotetext{
“llenamos el pizarrón de listas de palabras que a ellos les parecían lindas (pájaros, sol, agua, canto, arboles, sombra, fresco, etc.). Otra lista con verbos (cantan, vuelan, construyen, alegran) y otra con adjetivos. Construyeron oraciones que me sorprendían, utilizando algunas de las palabras que había escrito en la pizarra y que ellos me iban dictando. Esta clase sirvió muchísimo para en las siguientes reconocer sujeto, predicado, complementos, etc." (María, docente).
}

En este fragmento, se observa como partió de los sentimientos y pensamientos de los estudiantes para enseñarles contenidos que estaban en el programa lo que implica cierta "superación" de la cosificación y verticalidad a la que están sujetos aquellos en la pedagogía "bancaria” (Freire, 1999).

Asimismo, se presenta un ejemplo donde la maestra tiene en cuenta las condiciones materiales de existencia de sus estudiantes para elaborar los contenidos, aunque no lo realiza, en forma dialógica, como afirma Freire que debe ser una pedagogía del oprimido, sino a partir de su propia visión del mundo. En este sentido, afirma que,

“elegí hacerme cargo de enseñar huerta en las tareas co-programáticas por considerar que era muy útil para estos chicos, que se interesaran por hacerla en sus casas (...) cuando se cortaba los niños llevaban a sus casas un poquito de esa verdura” (María, docente). 
En consonancia con los enfoques interaccionistas, para Freire, el oprimido termina siendo lo que el opresor le dice que es. En este sentido, sostiene que terminan interiorizando la visión que tienen los opresores de ellos dando como resultado la autodesvalorización de los oprimidos. Así, afirma que,

\footnotetext{
"de tanto oír de sí mismos que son incapaces, que no saben nada, que no pueden saber, que son enfermos, indolentes, que no producen en virtud de todo esto, terminan por convencerse, de su "incapacidad". Hablan de sí mismos como los que no saben y del profesional como quien sabe y a quien deben escuchar. Los criterios del saber que les son impuestos son los convencionales" (Freire, 1999, p. 13).
}

El interaccionismo simbólico es una corriente sociológica desarrollada por la Escuela de Chicago, específicamente por George Mead, en la década del treinta que pone en el centro del análisis la perspectiva del actor. Esta corriente parte de estudiar al individuo como un ser que actúa según la interpretación que hace de los objetos, situaciones y acciones de los otros. De este modo, para comprender la conducta de un individuo se debe estudiar como el sujeto ha interpretado la situación en base a la cual siguió su curso de acción es decir la perspectiva del actor. En este sentido, es importante el modo en que definen los actores las situaciones ya que actúan en función de dicha definición.

Así, si los hombres definen una situación como real ésta es real en sus consecuencias. De este modo, la percepción que las personas tienen de los otros y de la situación, influye sobre el otro y en la situación. De esto deriva que el concepto que tengan los docentes sobre los alumnos influye sobre estos generando una especie de "profecía que se cumple a sí misma" ya que las personas tienden a actuar de acuerdo a como los definen los demás. Esta definición que realizan unos acerca de otros muchas veces funciona como una "etiqueta" que hace que el individuo se convierta en lo que la etiqueta dice que es.

A partir del relato de la maestra, se puede ver como utiliza ciertas "etiquetas": "alumnos más capaces", "alumnos que dan más trabajo", "alumnos que no hacen nada", etc. Sin embargo, no podemos saber si las mismas surtieron algún efecto en los comportamientos de los estudiantes. A pesar de esto podemos deducir algunas consecuencias a partir de los aportes que realiza el interaccionismo simbólico a los estudios socioeducativos.

Uno de los efectos más importantes de estas etiquetas puede ser el "efecto halo". Esto implica que el docente en base a aspectos muy reducidos de su personalidad define al alumno de forma positiva o negativa extendiendo aquellos rasgos a toda su persona: aquellos que se adaptan a sus expectativas son definidos de forma positiva y aquellos no de forma negativa. En este sentido, el hecho de calificar a un alumno como "trabajoso", "capaz", "incapaz", "vago", etc., implica que haber tomando solo un rasgo de su individualidad como ser su desempeño en la escuela, en determinada clase, bajo determinadas circunstancias, como algo que define completamente su personalidad y contribuir, de este modo, a que el estudiante termine comportándose como tal.

Sin embargo, no siempre estos aceptan las etiquetas, sino que también pueden resistirse a ellas. En este sentido, cabe destacar que, en el caso concreto de los estudiantes que mas trabajo de "disciplina" daban, la maestra a pesar de utilizar esta etiqueta no los estigmatiza marginándolos sino, por el contrario, conteniéndolos y brindándoles afecto, lo que puede llevar a suponer que dichos alumnos no asumieron la identidad que se les daba en tanto la maestra afirma que "encontró en el afecto una forma de hacerle llegar el conocimiento".

\section{CONCLUSIÓN}

A través de este trabajo se pudo observar como una misma práctica docente puede ser analizada desde distintas perspectivas sociológicas acerca de la educación. Esto nos da la idea de que las experiencias no son del todo coherentes, sino que están llenas de contradicciones. Así, por ejemplo, una misma maestra 
puede llevar adelante acciones que responden a una concepción funcionalista de la educación, como por ejemplo "imponerles" aquellos temas que en un diario son importantes, así como también algunas que se "corren" de esta perspectiva y aparecen más cercanas a la pedagogía del oprimido, como por ejemplo tener en cuenta aquellas palabras que a los estudiantes les parecen “lindas" para enseñarles lengua.

Sin embargo, más allá de estas contradicciones, se sostiene que esta docente tiene una visión del hecho educativo más cercana a la perspectiva funcionalista que a cualquier otra que impacta en sus prácticas. Seguramente esto se debe a que se trata de una maestra formada en una escuela "normal". Esta tradición en Argentina se ubico dentro de las estrategias del Estado para "homogeneizar" o "civilizar", a la sociedad que se presentaba heterogénea a causa de las migraciones y de la población nativa. En este sentido, para la conformación del Estado Nación (1880) era necesario homogenizar la lengua, la cultura, la ideología. Había que disciplinar. Así, la escuela cumplió un rol fundamental.

En este contexto, el docente era visto como el difusor la cultura, pero una cultura referida a los comportamientos y a los conocimientos básicos necesarios para la mayoría de la población. Davini va a decir que "este saber ha estado expresado en forma de normas universalistas, formulas, valores y principios requeridos para actuar en el nuevo "orden"” (Davini, 1995, p.22). El docente se encaraba de llevar a cabo las campañas de salud pública y diversas acciones de control social y es aquí en donde se va a poner la mirada. Así, por ejemplo, la docente cuenta en el relato que ella iba casa por casa a ver como vivían sus alumnos. Esto si bien produce admiración por la dedicación de la docente puede llegar a estar cruzado por la tradición normalizadora bajo la que ella se formó. También cuando usa la frase de que los estudiantes en las actividades de geometría no se "indisciplinaban" se puede notar que en su formación la búsqueda de lograr disciplina en los estudiantes era un objetivo muy relevante.

Asimismo, como continuidad y correlato de la educación familiar, en este contexto, la ocupación fue rápidamente definida como femenina dentro de un discurso tutelar: apoyo, ayuda, segunda madre en el marco de un sistema patriarcal. En el presente análisis esto es clave ya que en los fragmentos se puede ver como la docente trata de brindarle afecto a sus estudiantes, como trata de consentirlos, ayudarlos como lo hace una madre. Asimismo, se observa como ella misma dice que todos los comienzos de año iban casa por casa a ver quienes eran sus estudiantes, cómo vivían, mientras los mismos tenían actividades con las maestras especiales o salía antes de su casa. Aquí se ve una entrega personal, al igual que cuando hace de confidente de las mamás de los estudiantes.

Más allá de las limitaciones del artículo, especialmente que se centra en la experiencia de una sola docente, se cree que aporta elementos valiosos para comprender la visión del hecho educativo de las maestras "normales". Asimismo, genera nuevas preguntas, como por ejemplo: ¿la experiencia de María se generalizable a la de todas las maestras formadas en escuelas normales de Argentina de comienzos del siglo XX? ¿Su visión del hecho educativo, ligada específicamente al funcionalismo, expresa el modo en que fue educada? ¿Se podrán encontrar experiencias y visiones alternativas en la docencia argentina de aquellos años?

\section{REFERENCIAS}

Altamirano, R. (2018). El proceso de oficialización de la docencia: Escuela Normal Argentina. Sociales y Virtuales, 5(5). http:// socialesyvirtuales.web.unq.edu.ar/el-proceso-de-oficializacion-de-la-docencia

Bourdieu, P., y Passeron, J. (1977). La reproducción. Elementos para una teoría del sistema de enseñanza. Editorial Laia.

Bourdieu, P. y Wacquant, L. (2008). Una invitación a la Sociología reflexiva. Siglo XXI Editores.

Bronckart J.P y Schurmans, M.N. (2006). Pierre Bourdieu-Jean Piaget: habitus, esquemas y construcción de los psicológico. En B. Lahire, El trabajo sociológico de Pierre Bourdieu. Deudas y críticas, (pp. 181-206). Siglo XXI.

Coulon, A. (1995). Etnometodología y educación. Editorial Paidós.

Davini, M.C. (1995). La Formación Docente en cuestión: Política y pedagogía. Editorial Paidós.

Dreeben, R. (1999). La contribución de la enseñanza al aprendizaje de las normas. En, M. F. Enguita. Sociología de la Educación, (pp. 512-533). Editorial Ariel. 
Durkheim, E. (1975). La educación: su naturaleza y su papel. En E. Durkheim, Educación y Sociología, (pp.43-49). Editorial Península.

Durkheim, E. (2003). Las reglas del método sociológico. Prometeo Libros.

Durkheim, E. (2008). El suicidio. Editorial Losada.

Fenstermacher. G. (1989). Tres aspectos de la filosofía de la investigación sobre la enseñanza. En M. Wittrock. La investigación de la enseñanza, (pp. 149-159). Editorial Paidós.

Freire, P. (1999). Pedagogía del Oprimido. Siglo XXI.

Gómez, M. (2016). La disciplina escolar y los procesos intersubjetivos entre docentes y alumnos. Mimeo.

Gvirtz, R. y Palamidessi, M. (2000). El ABC de la tarea docente: currículo y enseñanza. Aique.

Lahire, B. (2008). Cultura escolar, desigualdades culturales y reproducción social. En E. Tenti Fanfani. Nuevos temas en la agenda de la política educativa, (pp. 35-52). Siglo XXI.

Ouviña, H. (2002) El Estado: su abordaje desde una perspectiva teórica e histórica. En S. Lifszyc. (comp.). Introducción al conocimiento de la Sociedad y el Estado. Gran Aldea Editores.

Parsons, T. (1976). Las funciones sociales de la clase escolar. En A. Grass (comp.). Sociología de la educación. Textos fundamentales, (pp. 55-60). Narcea.

Ranciere, J. (2007). El maestro ignorante. Cinco lecciones sobre la emancipación intelectual. Libros del Zorzal.

Rist, R. (1999). Sobre la comprensión del proceso de escolarización: aportaciones de la teoría del etiquetado. En F. Enguita. Sociología de la Educación, (pp. 615-627). Editorial Ariel.

Tenti, E. (1994). La educación como violencia simbólico. En G. González Rivera y C. Torres (comp.). Sociología de la educación. Corrientes contemporáneas, (pp.7-39). Miño y Dávila.

\section{AUTORA}

María Dolores Sancho. Licenciada en Sociología de la Universidad de Buenos Aires. Magíster en Ciencias Sociales y Humanidades por la Universidad Nacional de Quilmes y Doctoranda en Ciencias Sociales en la Universidad de Buenos Aires. Docente e investigadora de la Facultad de Derecho y Ciencias Sociales de la Universidad Nacional del Comahue.

\section{Conflicto de intereses}

La autora informa de ningún conflicto de interés posible.

Financiamiento

No existió asistencia financiera de partes externas al presente artículo.

Agradecimientos

A mi hermana incondicional, Celeste y a mi abuela eterna, Marilo. 